\title{
Design of Remote Virtual Experiment Based on MATLAB and .NET
}

\author{
Mu Xianmin ${ }^{\mathrm{a}}$, Chen Xiyou ${ }^{\mathrm{a}}$, Li Meifang ${ }^{\mathrm{a}}$, Li Guanlin $^{\mathrm{a}}$ \\ ${ }^{a}$ School of Electrical Engineering, Dalian University of Technology, Dalian, China
}

\begin{abstract}
This paper presents a framework for remote virtual experiment implementation. The work principle of the solution is described and the three-tier structure of the system is proposed. The proposed solution is based on two commercially available software packages. MATLAB/SIMULINK is used for experiment module development, while .NET is used for the user front-end interface. The virtual experiment is divided into two kinds, static one and dynamitic one. The static remote virtual experiment is designed by MCR interface and the dynamitic one is done by MATLAB engine technique. In addition, two samples of remote virtual experiment are deployed to user.
\end{abstract}

Index Terms: Remote Virtual Experiment; Dynamitic Experiment; Static Experiment; Three-tier

(C) 2011 Published by MECS Publisher. Selection and/or peer review under responsibility of the International Conference on E-Business System and Education Technology

\section{Introduction}

It is the effective method that students can take on research or rebuild through experiment course. Because of the lack of lab models or real physical systems, and the little availability of schedules in the laboratories where the students of automatics and process control subjects develop the practical assignments corresponding with the theoretical contents.

However, at present, for the some foundation courses focus on teaching, the experimental courses are limited by the laboratory equipment, time and space. It causes that the progress of experimental course commencement cannot maintain consistent with the progress of theory course. Sometimes theory courses ever have finished, the experiment did not start yet. Experimental class as supplement of theory class cannot achieve the intended effect, and teacher cannot interact with the students timely and effectively in the teaching process too.

With the improvement of modern computer simulation technology, experiments in the teaching process specialized simulation software can overcome some constraints in laboratory[1-3]. Yet, due to copyright issues such as professional software, it is impossible to access to this software environment for students at any time.

Therefore, the presented application is focused on the remote virtual experiment through Internet and MATLAB as teaching and development platform. It will allow the students to simulate experiment through 
Internet. In this way, the student is allowed to develop these activities without being in the laboratory. By developing a virtual experiment platform, more efficient network experiment environment extended from the traditional laboratory is created for teachers and students, which is more relaxed in the time and space and without restrictions in application.

MATLAB is mathematical software developed by MathWorks Company, with the matrix operation and numerical analysis and scientific computing functions, .NET is a network solution platform developed by Microsoft, with the advantages of uniform interface and easy to use. .NET is flexible to use and MATLAB is good at calculating. Combining both advantages, it is supposed to establish a simulation model of experiment by using MATLAB to achieve computing and data processing functions, and integrate in NET application, It can maximize the advantage of both platforms [4-6]. With the use of MATLAB and .NET technology, this paper uses three-tier structure, fully exert respective advantages, establish electrical technology network virtual experimental system, realize specific network virtual experimental subject.

\section{Structure of Remote Virtual Experiment}

The virtual experiment technology using software simulation method to establish the experimental simulation model, through the simulation calculation, obtain the data and curves of the simulation results of the object model, it can achieve the same effect as the actual experiment. Remote virtual experiment is a technology that multiple users access to a virtual experiment server through the network. The remote virtual laboratory generally uses server and client mode and its function diagram is shown in Fig. 1.

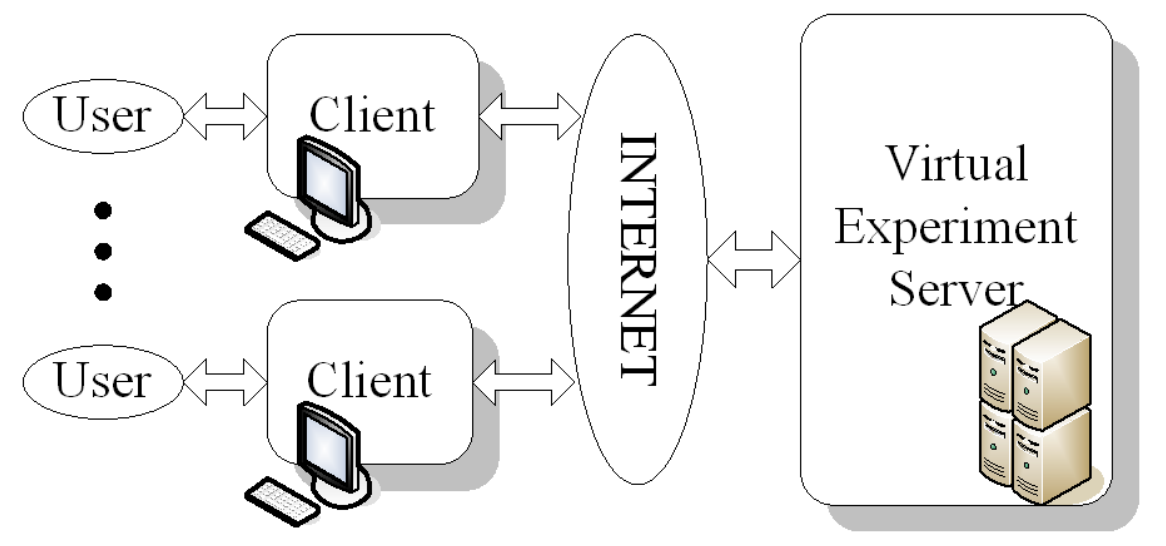

Fig. 1. Structure of remote virtual experiment

The virtual experiment server installed software for simulation and calculation, which mainly provide simulation model established, simulation, computing and network services function; client provides interactive function for the user and the virtual experiment server to transfer data between each other, so users enable to access remote virtual experiments via internet.

The same as general providing network service systems, according to the different forms of client, the virtual experiment system is divided into two categories, namely: fat client virtual experiment system and thin client virtual experimental system[7,8], corresponding to the C/S (Client / server) mode and B/S (Browser / Server) mode. For the thin client system, students do not need to assemble large-scale virtualization software, simply use of campus network and PC terminals, install the browser, they can communicate with the server configured and equipped with virtual experimental software, proceed online virtual experiment activities through the user interface. Thin client system is simple to use, high flexibility and maintainability. Therefore, this article adopts thin-client model to develop remote virtual experiment system. 
Thin client network service system, there are two B / S platform is available, the J2EE platform and. NET platform. J2EE platform based on Java language, use Java2 platform to simplify the solution; offer interlayer integration framework; provide a unified development platform; open standards. . NET platform shorted for Microsoft.NET platform, is a multi-language component development and execution environment, supports various languages (such as $\mathrm{VB}, \mathrm{VC}, \mathrm{VC}++, \mathrm{VJ}$, etc.) ease of use.

Take the demand of the system, usability, cost and scalability, etc. into account, this paper adopts .NET as the development platform of virtual experiment system. Virtual experiment underlying source code software adopts widely used and professional software MATLAB.

\section{Realization of Remote Virtual Experiment}

The remote virtual experimental system can be divided into three layers structure according to its function. As shown in Fig. 2, it includes display logical section (Presentation layer), things processing logical section (Interface layer) and file management section (File layer). The function of Presentation layer is bring about the interaction with the user; the function of Interface layer is converted the user requests to specific operation and data processing; the function of File layer is satisfy the desires of data stored, data read and update operation.

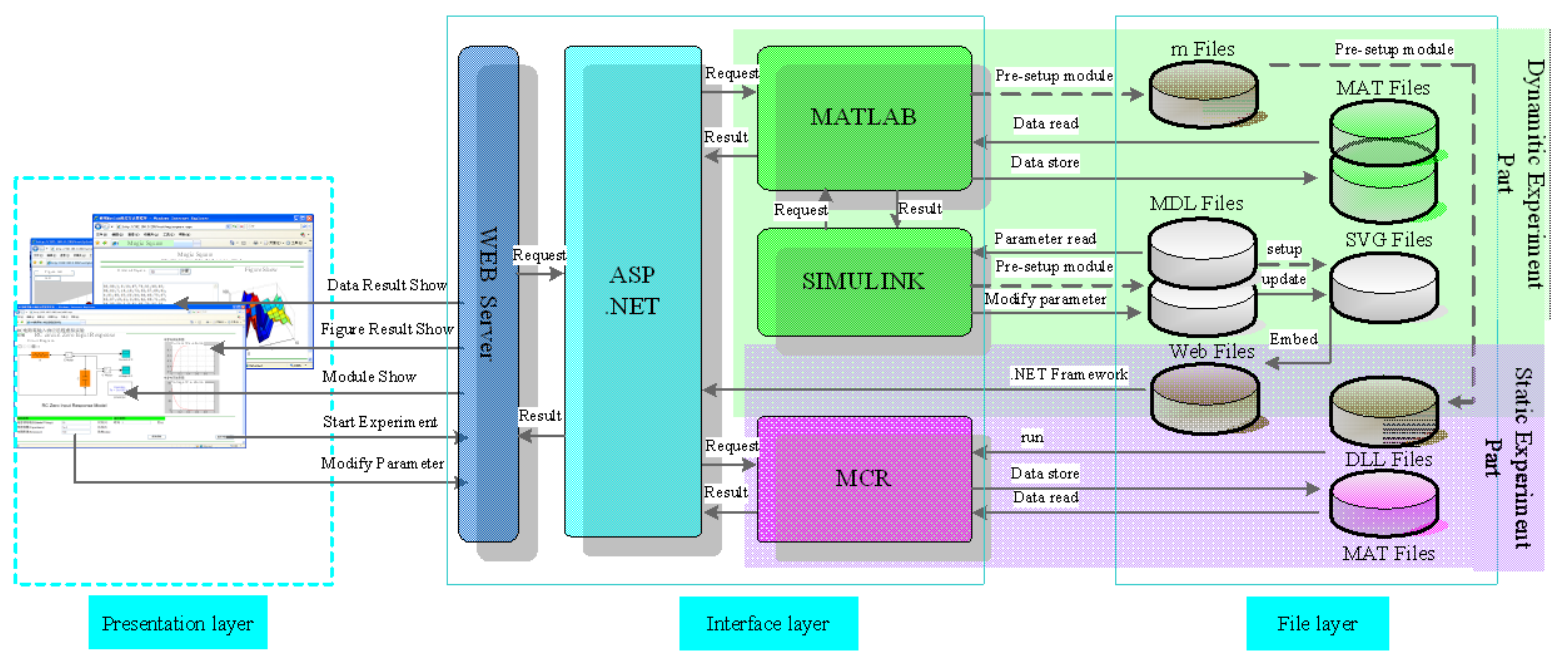

Fig. 2. Three-tiers of remote virtual experiment

The experiment is divided into two categories: Dynamic experiment and static experiment. Dynamic experiment refers to that the experiment result data contains time values. It needs to use SIMULINK to establish dynamic model; Static experiment refers to that the experiment data is not. It can be achieved using $m$-file of MATLAB easily.

The required resource of dynamic experiment and static experiment are in the upper shadow part and the lower shadow part in file layer and interface layer respectively. From the block diagram, the simple static experiment can be supported by the MCR (MATLAB Component Runtime). MCR will analytical $m$ files of MATLAB into the supported CLR (Common Language Runtime). For dynamic experiments need MATLAB and SIMULINK to support.

Therefore when to set up the server, virtual experiment system to the server configuration can be adopted to use 3 (group) servers to accomplish, it is realized respectively by WEB service, the function of dynamic experiment and static experiment. As shown in Fig. 3 of virtual experimental server configuration diagram, users can visit through the Internet or access inside campus network. 


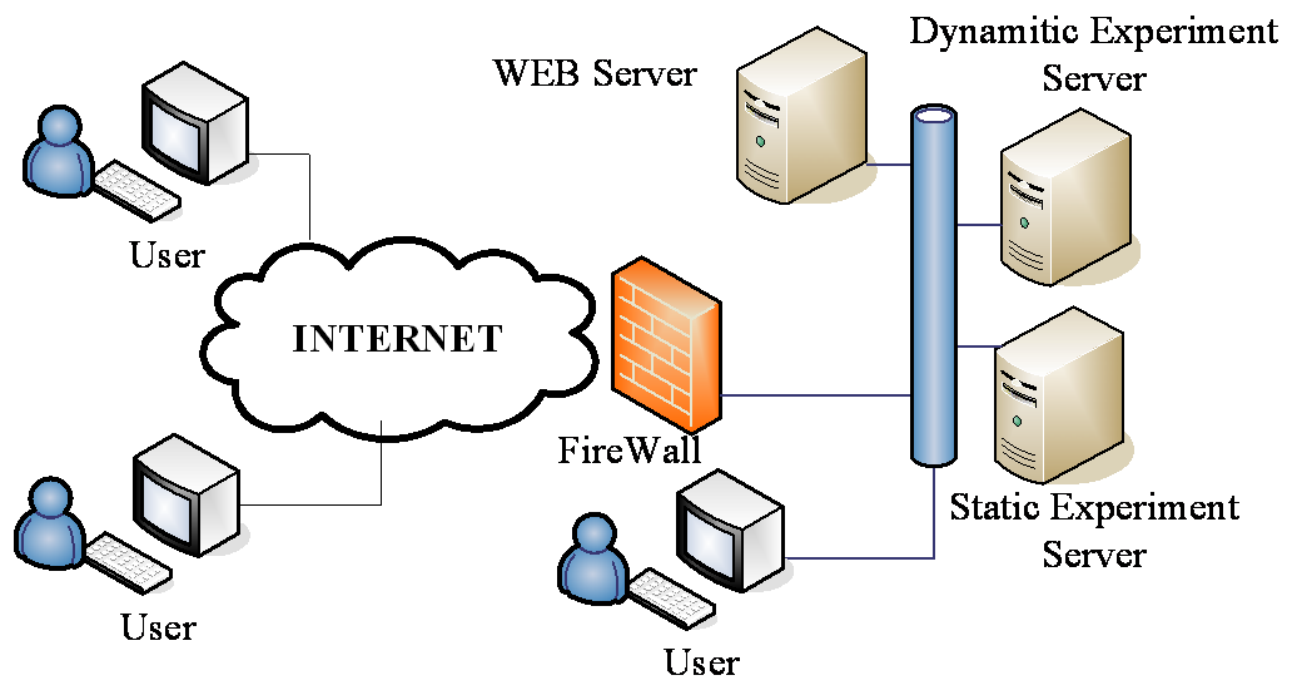

Fig. 3. server configuration of remote virtual experiment

\subsection{Resources and Function Description of Three-tier}

\section{1) Presentation Layer}

Also known as a client, it is the browser, which implements interactive features with the specific virtual experiment system. The browser is responsible to receive user's requests, and then send the requests to the WEB server of interface layer, and get the desired results from the WEB server, meanwhile displayed them in the browser. The results including two forms of data and pictures are back to the user, who can save the data or images locally according to user's need. The client with the function of login system, users can choose to experiment, set experiment parameters, start experiments, display experimental results and other specific action.

\section{2) Interface Layer}

It is responsible for interface layer to turn the user's requests into the corresponding underlying simulation software of concrete operation. It mainly include WEB server, ASP.NET engine, MATLAB, SIMULINK, and MCR several subsystems. In which, WEB server receive requests from the user then pass it to the ASP.NET subsystem, and transmit the results obtained from the ASP.NET to client browser; ASP.NET subsystem parse web documents responsibly, and sent it to WEB server as needed, for the use of browser. If it is dynamic experiments, the web documents will passed to MTLAB for calculated, and static experiment, they will transferred to MCR. For dynamic experiments, MATLAB need starting SIMULINK to simulate and calculate.

Interface layer is the core of the virtual laboratory system, but also has functions of providing fast and secure network connections, user authentication, and acceptance test requests of client, store test result data, and analysis experimental data.

\section{3) File Layer}

File level support the provision of basic data structures for the virtual laboratory system. It mainly includes Web aspx files, MATLAB function $m$ files, SIMULINK simulation model $m d l$ files, simulation models show $s v g$ files, MATLAB data mat files and dynamic link library dll files. Static experiments only contain $m$ files, aspx files, mat files and $d l l$ files (which $d l l$ files include dynamic-link library documents of two types, generated by $m$ files or Web publishing); dynamic experiments contain the mdl file, aspx file, svg file, mat file and dll file. 
When establishing the virtual experiments, it is necessary to pre-built $m$-files and $m d l$ files first. The $\mathrm{m}$ function file after the formation of MATLAB will transform into the dll files that support for parameter input, user can modify the parameters of static experiments; using the SIMULINK command, parameters in $m d l$ file can be set, so the functions of modified parameters in dynamic experiment realize.

\subsection{Software Realization of The Three Layer Structure}

The software of virtual experiment system is mainly composed of MATLAB and NET, Therefore the good performance of the network interface for the effective operation of virtual experiment system has important significance. The main interface form of .NET and MATLAB has the following kinds:

\section{1) Cor C++ interface}

MATLAB compiler can convert the MATLAB $m$ files to $\mathrm{C}$ or $\mathrm{C}++$ code, and it can carry out without MATLAB independently.

\section{2) Component technology of the .NET or COM}

COM, namely Component Object Model, allows applications use MATLAB by different software products. MATLAB Builder for .NET, it can generate alone .NET classes or COM objects from the MATLAB algorithm code.

\section{3) MATLAB engine technology}

MATLAB engine function library is a series of procedure provided by MATLAB, which allows users to carry out MATLAB command in their applications. MATLAB runs in the background as an engine.

The (1) kind of interface methods converts MATLAB scripts into $\mathrm{C}$ or $\mathrm{C}++$ language. SIMULINK $m d l$ model file also supports generating $\mathrm{C}$ or the $\mathrm{C}++$ language, so this method can satisfy the interface to the static and dynamic experimental realization, but it is not facilitate to modify and maintain them.

Therefore, as the static experiment of virtual experiment, we use the (2) kind of interface technology; while the dynamic experiment of virtual experiment, use the (3) kind of interface technology, which can direct call MATLAB instructions.

\section{Design Instances of Virtual Experiment}

Development environment of virtual electrical experiment platform use Asp.NET technology, with Microsoft Visual Web Developer as a development tool, the ASP.NET platform program is written in C\# language. Web server platform use IIS6.0 of Microsoft company. Take the Thevenin Equivalent circuit experiment and Zero-Input Response of RC circuit experiments as example; the remote virtual electrical experiments platform is designed.

\subsection{Experimental Function Analysis}

Thevenin Equivalent Circuit experiment, the experimental procedure is to obtain equivalent circuit parameters, verify and make the equivalent circuit instead the output of the original circuit. It is designed in accordance with the method of static virtual experiment. It includes setting and changing parameter of the original circuit, obtaining parameters of equivalent circuit, setting the load parameter, gaining the load voltage and so on.

Zero-Input Response of RC circuit experiment, the experimental process is to obtain Zero-Input Response curve of RC circuit, so it is designed in accordance with the method of dynamic virtual experiment. It includes the circuit parameter changing and setting, the response curve drawing functions and so on. 


\subsection{Make Experiments Model File and Test}

According to Thevenin Equivalent Circuit test, $m$ function file is prepared to complete the function that calculate equivalent circuit parameters, the original circuit output voltage under load and the output voltage of equivalent circuit under load. Input parameters of $m$ function file is the circuit component parameters, output is calculated results. It is carried out and verified in MATLAB at first. Then release into dll files for .NET using MATLAB deploy tool.

SIMULINK simulation models are established according to the RC Zero-Input Response circuit model. The $m d l$ files were also established and tested in SIMULINK, output curve data is stored in the workspace by setting file parameters. At the same time svg file is generated for display using output function of SIMULINK.

\subsection{Prepare Code and Test}

According the function, the web pages are layout, divided into parameter setting, the results displaying and experiments starting, and related background code is compiled. Background code complete display file of data and model by using .NET and MCR or parameters and instructions transferred between MATLAB and .NET. Finally, run and test the website pages.

\subsection{Publish Site}

Website is deployed to the server after function test. Interfaces are shown in Fig. 4 and Fig. 5 as follows.

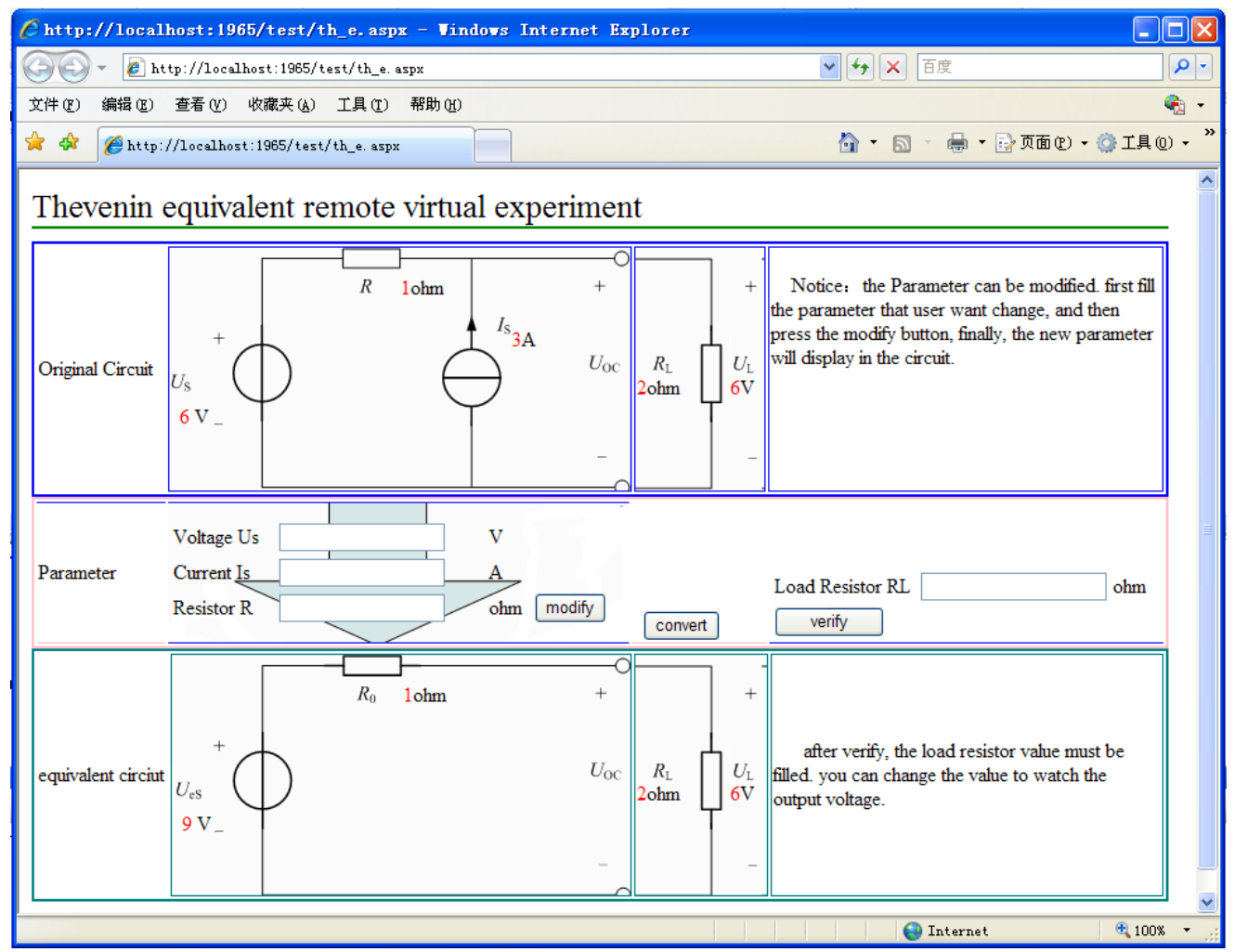

Fig. 4. Thevenin equivalent remote virtual experiment interface 


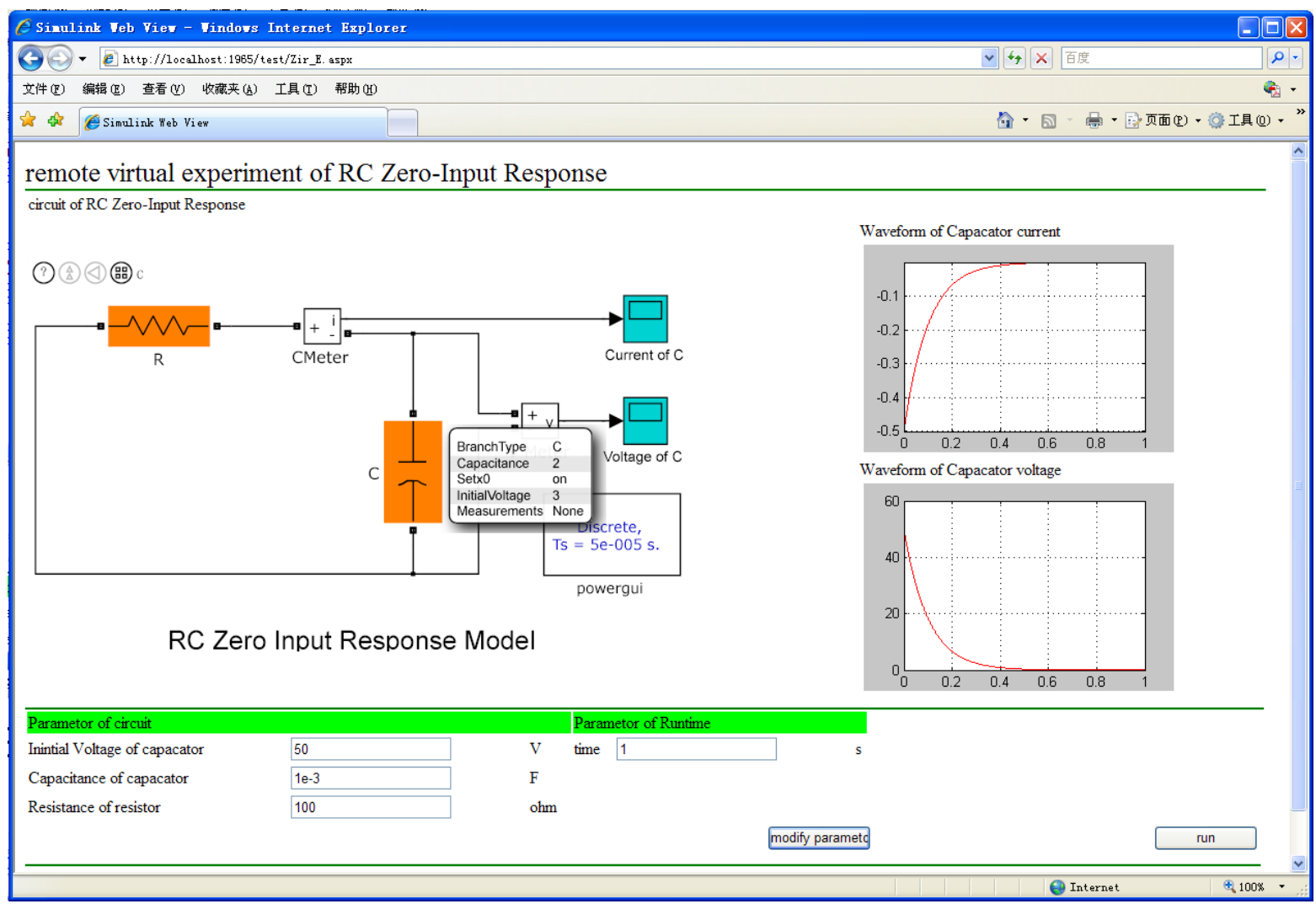

Fig. 5. remote virtual experiment interface of RC Zero-Input Response

As the friendly interface of virtual experiment system, prompting message perfect, the authorized user can finish the experiment topic very easy according to the tips of experiments interface.

\section{Conclusion}

Using Microsoft .NET technologies and MATLAB software, remote virtual experiment system is built, and we achieved experimental static virtual experiment and dynamic virtual experiment subject. Virtual experiment system takes full advantage of the campus network; overcomes the constraints of classroom teaching in time and space, solve the contradiction that knowledge expansion and the limited course hours in the traditional teaching model. It provides students with more extensive learning resources and study way, and meets the needs of individualized learning.

Now, the core part of virtual experiment of virtual experiment system is realized. Many parts will be increased, such as, information dissemination of experiment, experiment report management, results statistical evaluation, experiment doubts, the experiment related background information and other modules. It will constitute impeccable virtual experiment system. 


\section{References}

[1] Uran. S, Jezernik. K, "Virtual Laboratory for Creative Control Design Experiments," IEEE Transactions on Education, Vol: 51, Issue: 1, 2008 , p.69-75

[2] Menendez. L.M, Salaverria. A, Mandado. E, Dacosta. J.G, "Virtual Electronics Laboratory: A new tool to improve Industrial Electronics Learning," IEEE Industrial Electronics, IECON 2006 - 32nd Annual Conference on. 2006, p.5445-5448

[3] Hercog. D, Gergic. B, Uran. S, and Jezernik. K, “A DSP-Based Remote Control Laboratory," IEEE Transactions on Industrial Electronics, Vol: 54 , Issue: 6, 2007 , p.3057-3068

[4] Lingyun Li, Haijun Wang, "A New Method for Building Web-based Virtual Laboratory," Information Technologies and Applications in Education, 2007, p.555-558

[5] Prieto-Blazquez. J., Arnedo-Moreno. J, Herrera-Joancomarti. J, “An Integrated Structure for a Virtual Networking Laboratory,” Industrial Electronics, IEEE Transactions on. Vol: 55 , Issue: 6, 2008, p.23342342

[6] Uran. S, Hercog. D,Jezernik. K, "MATLAB Web Server and Web-based Control Design Learning." IEEE Industrial Electronics, IECON 2006 - 32nd Annual Conference on, 2006 , p.5420-5425

[7] Shoucheng Ding, Wenhui Li, Shizhou Yang, Jianhai Li, Wanqiang Lu, "The Motor Virtual Experimental System Based on Matlab Web Technology," Information Engineering(ICIE), 2010 WASE International Conference on Vol:1,2010, p.338 -341

[8] Weicheng Xie; Xia Yang; Fugan Li, "A virtual laboratory platform and simulation software based on web," Control, Automation, Robotics and Vision, 2008. ICARCV 2008. 10th International Conference on, 2008, p.1650-1654 